\title{
Alterstice
}

Revue internationale de la recherche interculturelle

International Journal of Intercultural Research

Revista International de la Investigacion Intercultural

\section{Remettre la culture à sa place pour mieux vivre ensemble : un exemple en milieu HLM à Montréal}

\section{Karoline Truchon}

Volume 1, numéro 1, 2011

Penser la recherche interculturelle : le défi des diversités

URI : https://id.erudit.org/iderudit/1077591ar

DOI : https://doi.org/10.7202/1077591ar

Aller au sommaire du numéro

Éditeur(s)

Alterstice

ISSN

1923-919X (numérique)

Découvrir la revue

Citer cet article

Truchon, K. (2011). Remettre la culture à sa place pour mieux vivre ensemble : un exemple en milieu HLM à Montréal. Alterstice, 1(1), 55-68.

https://doi.org/10.7202/1077591ar

\section{Résumé de l'article}

Près de $70 \%$ des résidents des HLM de Montréal sont maintenant des personnes issues de l'immigration. D'homogènes, les HLM de la métropole sont devenues dans la dernière décennie le lieu d'habitat des populations immigrantes, créant ainsi des incompréhensions et des tensions entre des Québécois nés ici et certains nés ailleurs. Les organismes qui travaillent en HLM tentent de rejoindre les populations dites " ethnoculturelles " ou " immigrantes » en implantant des projets qui favoriseraient la (re)connaissance des diverses ethnicités des résidents des HLM. Parmi eux, un organisme d'intervention psychosociale se distingue en réussissant à rejoindre et rallier les résidents de diverses origines ethnoculturelles sans travailler de manière délibérée sur l'axe de la culture. Il s'agit ici non pas de faire le procès de la culture et d'annoncer sa fin, mais plutôt de la remettre à sa place : en abordant le concept de manière transversale et non frontale, on faovriserait l'intégration des populations dites immigrantes, ainsi que le vivre-ensemble de la communauté où elles vivent. 


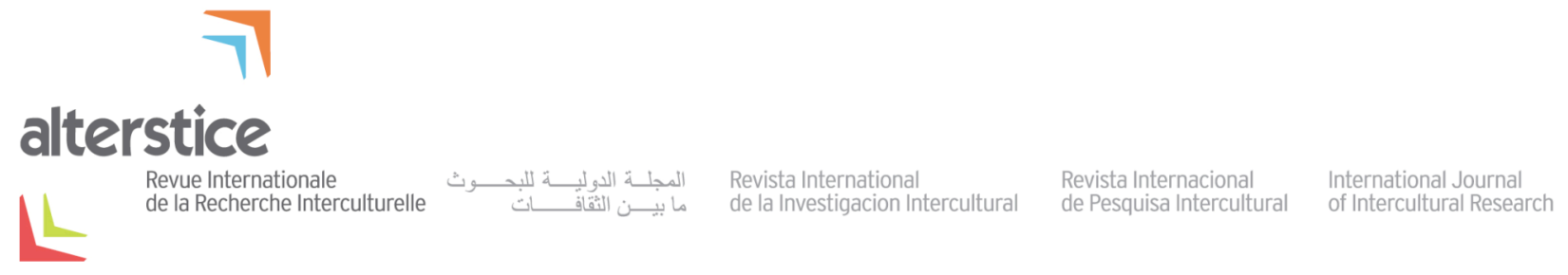

ARTICLE THÉMATIQUE

\section{Remettre la culture à sa place pour mieux vivre ensemble : un exemple en milieu HLM à Montréal}

Karoline Truchon ${ }^{1}$

\section{Résumé}

Près de $70 \%$ des résidents des HLM de Montréal sont maintenant des personnes issues de l'immigration. D'homogènes, les HLM de la métropole sont devenues dans la dernière décennie le lieu d'habitat des populations immigrantes, créant ainsi des incompréhensions et des tensions entre des Québécois nés ici et certains nés ailleurs. Les organismes qui travaillent en HLM tentent de rejoindre les populations dites " ethnoculturelles" ou "immigrantes» en implantant des projets qui favoriseraient la (re)connaissance des diverses ethnicités des résidents des HLM. Parmi eux, un organisme d'intervention psychosociale se distingue en réussissant à rejoindre et rallier les résidents de diverses origines ethnoculturelles sans travailler de manière délibérée sur l'axe de la culture. Il s'agit ici non pas de faire le procès de la culture et d'annoncer sa fin, mais plutôt de la remettre à sa place : en abordant le concept de manière transversale et non frontale, on faovriserait l'intégration des populations dites immigrantes, ainsi que le vivre-ensemble de la communauté où elles vivent.

\section{Rattachement de l'auteure}

${ }^{1}$ Département d’anthropologie et CÉLAT, Université Laval, Québec, Canada.

\section{Correspondance}

karoline.truchon.1@ulaval.ca

\section{Mots clés}

vivre-ensemble; reconnaissance; culture; HLM

\section{Pour citer cet article :}

Truchon, K. (2011). Remettre la culture à sa place pour mieux vivre ensemble: un exemple en milieu HLM à Montréal. Alterstice, 1(1), 55-68. 


\section{Introduction}

Le lieu est sacré quand il fait lien, mais c'est le lien qui fait le lieu et non l'inverse.

Régis Debray

Les organismes qui travaillent en milieu HLM (habitations à loyer modique) à Montréal tentent de rejoindre les populations dites "ethnoculturelles ", "multiethniques " ou "immigrantes " en implantant des projets pour favoriser la reconnaissance des diverses ethnicités ou appartenances culturelles des résidents de ces HLM. Par exemple, depuis 1999, le projet " Habiter la mixité » de l'Office municipal d'habitation de Montréal (OMHM) tente de répondre aux besoins grandissants d'accueil et d'intégration des personnes nouvellement arrivées dans la métropole, ainsi que de celles ayant émigré depuis plus longtemps et qui emménagent dans des logements sociaux gérés par l'Office. Il montre l'importance de résoudre les questions liées à la cohabitation interculturelle, et constitue l'un de six enjeux et priorités de l'OMHM. II est implanté dans neuf HLM des cinq arrondissements couverts par l'Office.

Parmi ces organismes, l'un, le Centre des jeunes Boyce-Viau (CJBV), se distingue en réussissant à rejoindre et rallier des résidents d'un HLM nés à l'extérieur du pays sans travailler de manière délibérée sur l'axe de la culture. À partir de l'étude des modalités d'intervention et d'animation de cet organisme, ainsi que des modalités de recherche et d'implication en tant qu'anthropologue, l'objectif de ma recherche a été non de faire le procès de la culture et d'annoncer sa fin, mais plutôt de la remettre à sa place. En abordant la culture de manière transversale et non de manière frontale, on favoriserait sans doute mieux non seulement l'intégration des populations dites " immigrantes ", " ethnoculturelles " ou " multiculturelles », mais également le vivre-ensemble des collectivités où elles vivent en compagnie d'autres personnes nées au Canada. II faut souligner que le concept de culture en sciences sociales, ou plus précisément le «pluralisme culturel » en intervention psychosociale au Canada et au Québec, a la particularité d'orienter les débats publics et politiques en servant d'outil de gestion de l'identitaire (Armony, 2007a et 2007b; Beauchemin et Bock-Côté, 2007; Benessaieh, 2010; Bock-Côté, 2009 et 2007; Kymlicka, 2007a, 2007b et 2007c; Lachapelle, 2008; Lisée, 2007a et 2007b; Taché, 2009). Bien que cet article ne traite pas des concepts de multiculturalité, d'interculturalité et de transculturalité en tant que tels, il aborde la composante commune à chacun d'eux: la dimension culturelle, qui, selon chaque paradigme, est travaillée de manière différente et détermine une conception et une application différentes du concept de culture.

Avant d'aller plus loin avec cette proposition, je rappelerai le débat concernant l'apport du concept de culture pour les chercheurs travaillant avec des personnes en situation de pauvreté. Dans ce cadre, le concept de culture a été contesté dès ses premières utilisations dans les années 1960, puis a été négligé ou omis au sein des priorités de recherche contemporaines, et ce n'est que depuis quelques années qu'il vit une renaissance. Nous verrons comment des chercheurs intègrent dans leurs analyses des "dimensions culturelles » de personnes vivant en situation de pauvreté plutôt que de considérer la culture comme une situation homogène et monolithique partagée par toutes ces personnes. Nous constaterons également que ce qui est appelé une «culture de pauvreté " s'apparente plus à une "culture de l'inégalité ", car c'est au sein d'éléments structurels et environnementaux que les croyances sur lesquelles repose la culture de la pauvreté se développent et s'entretiennent.

J'enchaînerai ensuite avec une proposition visant à appréhender le culte voué à la " culture " en questionnant la validité de deux prémisses qui façonnent la pratique de l'anthropologie - discipline qui a choisi de se scientifiser en s'appropriant et en développant le paradigme de la culture - , soit la " différence » et la spatialisation de cette différence. L'idée que l'autre est nécessairement différent de soi et la relégation de cette différence à un espace fixe seront discutées dans le but d'envisager une nouvelle manière de saisir les réalités des personnes participant à des recherches, afin de ne pas les enfermer, sous couvert de la définition de "leur culture ", dans un carcan psychologique et spatialisé qui aurait plus à voir avec l'imagination et la validation d'une discipline qu'avec les réalités "empiriques » des personnes étudiées. II est difficile pour ces personnes de se défaire de ce carcan une fois que cette conception est véhiculée dans l'espace universitaire et public par les chercheurs et les médias.

Alterstice-Revue Internationale de la Recherche Interculturelle, vol. $1, n^{\circ} 1$ 


\title{
Débattre l'apport de la " culture » en situation de pauvreté
}

\author{
Whether we have free will or not, we certainly aren't completely free, autonomous individuals. \\ We influence and are influenced by our physical and social environments, often without our awareness.
}

Quinn O'Neill

Nous pourrions penser qu'un concept aussi utilisé, normalisé, voire banalisé, que celui de culture posséderait des balises assez précises en ce qui concerne sa définition et ses applications. Tel n’est pas le cas. La littérature scientifique abonde sur ce qu'est la culture sans jamais statuer définitivement sur sa définition. Dès les années 1950, Alfred Kroeber et Clyde Kluckhohn (1952, cités par Ferraro et Andreatta, 2010, p. 28) répertoriaient plus de 160 définitions de la culture, faisant de celle-ci un concept pluriel aux contours flous. Considérant qu'un consensus n'est pas près de survenir sur cette question, Mario Luis Small, David J. Harding et Michèle Lamont (2010), des sociologues travaillant avec des personnes en situation de pauvreté, préconisent, à l'instar d'autres chercheurs, de réintégrer autrement la variable culturelle dans les priorités de recherche contemporaines (Frerer et $\mathrm{Vu}, 2007)$. Ils proposent de conceptualiser l'aspect culturel des dynamiques sociales en déplaçant le regard d'analyse de "la culture " à celle de perspectives révélatrices d' "éléments culturels ", ou encore de saisir comment des dimensions et des facteurs culturels modèlent les réponses de personnes vivant en situation de pauvreté (Small, Harding et Lamont, 2010, p. 14). Ils justifient ainsi leur position, qu'ils jugent " pragmatique » : le concept de culture " cache plus qu'il ne révèle, du moins lorsqu'il s'agit de saisir un problème particulier ${ }^{1}$ " (Small, Harding et Lamont, 2010, p. 15, traduction libre). L'interrogation des chercheurs contemporains n'est donc plus de savoir si la culture provoque la pauvreté, mais d'anticiper quels facteurs culturels façonnent la compréhension et l'expérience de la pauvreté par les individus, et quelles réponses ils offrent à cette dernière (Small, Lamont et Harding, 2011). Sept dispositifs d'analyse jugés plus appropriés que le concept de "culture » émergent de leur réflexion: les valeurs (values), les cadres de référence (frames), les répertoires (repertoires), les narrations (narratives), les frontières symboliques (symbolic boundaries), le capital culturel (cultural capital) et les institutions (institutions) (Small, Harding et Lamont, 2010, p. 14-20).

Les valeurs indiquent les finalités recherchées par un comportement ( $p .14)$ et les cadres de références sont les lunettes à travers lesquelles une personne observe et interprète ce qu'elle est comme individu, ainsi que ce que sont le monde et l'environnement où elle évolue (p. 14-15). Les répertoires sont des actions régies par deux conditions : la première stipule qu'une personne possède une liste, un répertoire de stratégies et d'actions, et la seconde, qu'une personne ne sera pas encline à agir si la stratégie ou l'action ne font pas partie de son répertoire (p. 16). Les narrations sont possibles car les êtres humains interprètent leurs vies comme des histoires qui ont des débuts, des milieux et des fins. Ces histoires à plusieurs fragments narratifs existent grâce aux liens de causalité qui relient les fragments entre eux. Les narrations sont construites les unes en relation avec les autres, avec comme caractéristique de relier des parties du monde social plutôt que de simplement catégoriser. Formant des récits qui illustrent comment des individus se perçoivent en relation avec les autres, les narrations sont constitutives de la manière dont ils construisent leurs identités sociales. Les narrations imprègnent donc les actions des personnes, car celles-ci sélectionnent des actions qui sont consistantes avec leurs identités et narrations personnelles. Elles fascinent car elles font appel aux émotions et aux expériences humaines communes. De fait, elles illustrent les manières qu'ont les gens de donner du sens à leurs expériences, contraintes et occasions (Small, Harding et Lamont, 2010, p. 16-17). Les frontières symboliques sont les distinctions conceptuelles que les individus construisent entre les objets, les personnes et les pratiques. Elles fonctionnent comme un ensemble de normes érigé en un système de classification qui détermine la hiérarchisation de la valeur morale entre les individus et les groupes en comparant et jugeant les similarités et les différences entre eux (Small, Harding et Lamont, 2010, p. 17-18). Le capital culturel est défini comme étant « institutionnalisé, c'est-à-dire qu'il est un ensemble de signes

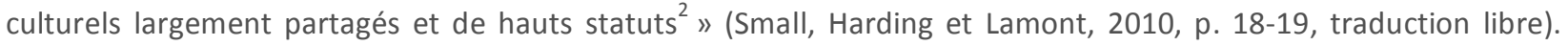
Finalement, les institutions forment trois entités: "as formal rules of behavior that are codified as laws or regulations, as norms of appropriate behavior that are enforced through informal sanctions, and as taken-forgranted understandings that simply structure or frame [...] how actors perceive their circumstances » (Scott, 1995, cité par Small, Harding et Lamont, 2010, p. 20). Ce déplacement de la " culture » à des " dimensions et facteurs

\footnotetext{
1 " ultimately masks more than it reveals, at least when the purpose is to understand a specific problem »

2 " institutionalized, i.e. widely shared, high status cultural signals »
} 
culturels " révélés par différents dispositifs favorise une autre manière d'appréhender le multi-culturel, l'interculturel et le trans-culturel, ouverture qui qualifie les modalités relationnelles intrinsèques à chacun des paradigmes et qui sera exposée dans la prochaine section.

Il est intéressant de constater que des sociologues (Small, Harding et Lamont, 2010) remettent en question l'un des paradigmes fondateurs de leur discipline, soit l'influence de la structure. Ils le font, nous venons de l'exposer, en proposant d'insérer des dimensions culturelles au sein de leurs analyses. Or la "culture " est, nous le résumerons dans la prochaine section, rattachée depuis le XIX ${ }^{\mathrm{e}}$ siècle à la discipline cousine, l'anthropologie. Cette incorporation de la culture dans les objectifs de recherche promus par Small, Harding et Lamont (2010) est néanmoins menée comme si la culture existait d'elle-même et par elle-même, en dehors de structures sociales, politiques, économiques et représentationnelles. En effet, ces chercheurs font primer les aspects culturels sur ceux structurels et, ce faisant, inversent le problème que nous soulevons de la « culture » au lieu de le régler :

Again we do not deny the importance of macrostructural conditions, such as the concentration of wealth and income, the spatial segregation across classes and racial groups, or the persistent international migration of labor and capital. Instead, we argue that since human action is both constrained and enabled by the meaning people to their actions, these dynamics should become central to understanding of the production and reproduction of poverty and social inequality. (Small, Harding et Lamont, 2010, p. 23)

De fait, malgré la conclusion à laquelle ils arrivent Small, Harding et Lamont, les sept dispositifs d'analyse d'éléments culturels qu'ils proposent sont influencés par les lieux, les organisations, les structures et les environnements. Par exemple, les répertoires sont des occasions et des contraintes qui influencent les décisions (p. 16); les narrations révèlent comment les personnes comprennent leurs expériences, contraintes et occasions (p. 16-17) et comment elles se saisissent des structures au sein desquelles elles évoluent qui en retour influencent elles aussi leurs expériences, leurs contraintes et leurs occasions; et le capital culturel est créé par la structure, l'environnement au sein duquel évoluent les individus (p. 18-19). Ainsi, en essayant de "réintégrer » la culture, on "désincarne " la structure, car je pense qu'au contraire d'être opposées, la culture et la structure sont complémentaires. La culture est créée à l'intérieur d'une structure et la structure est créée à l'intérieur d'une culture. La question pour nous est de maintenant mieux comprendre comment elles sont toutes deux médiées, individuellement, et intermédiées, entre elles. J'argumente donc que la culture et la structure doivent être appréhendées ensemble, pas comme de simples entités dont l'une prime sur l'autre, notamment l'action des êtres humains (human action) qui serait plus importante, comme le pensent Small, Harding et Lamont (2010), que l'aspect structurel de la société (structure), parce qu'au quotidien, elles agissent ensemble, en interdépendance, sans ordre précis. Elles constituent donc une économie morale dans le sens où l'entend Didier Fassin (2009, p. 1257) parce qu'elles articulent, ensemble, «la production, la répartition, la circulation et l'utilisation des sentiments moraux, des émotions et des valeurs, des normes et des obligations dans l'espace social » et que « les émotions ne sont pas séparées des valeurs et des normes ". Ainsi, les aspects plus subjectifs de la condition d'être humain sont constitutifs des aspects plus substantifs de cette condition et vice-versa.

\section{Décultifier et délocaliser la culture en éradiquant la politique de la différence}

Every individual is like all other human beings, like some other human beings, and like no other human beings. [...] Those attributes we share with some other human beings are cultural similarities.

Clyde Kluckohn

Des sociologues travaillant avec des personnes en situation de pauvreté ont donc formulé récemment une manière de réintégrer la dimension culturelle au sein des priorités de recherche contemporains par le biais de sept dispositifs d'analyse. Les anthropologues, bien qu'ils ne théorisent pas la pauvreté comme telle, peuvent contribuer à ce type de recherche par leur manière de théoriser, définir et contester la culture. Les définitions multiples de la culture, malgré leurs divergences, véhiculent que celle-ci est partagée, apprise et prise pour acquise (Ferraro et Andreatta, 2010, p. 30-35), contribuant à créer et cimenter une vision monolithique et incontestée, voire idyllique, romantique et a-politique de la culture. Or une compréhension des motifs ayant amené les anthropologues à établir la culture comme concept fondateur de la discipline nous amène à poser un regard plus critique sur celui-ci. D'une part, la culture comme concept principal de l'anthropologie a été sélectionné sciemment et de manière

Alterstice-Revue Internationale de la Recherche Interculturelle, vol. $1, n^{\circ} 1$ 
stratégique pour accomplir le rôle d'outil qui permettrait à la discipline de se crédibiliser sur le plan scientifique en devenant la "science de la culture", par exemple en calquant les modèles des scientifiques les plus respectés du $\mathrm{XIX}$ siècle, notamment les physiciens, les chimistes et plus particulièrement les biologistes, d'où la forte influence biométrique des débuts de la discipline (Ferraro et Andreatta, 2010, p. 201). Notre vocabulaire contemporain, certes teinté de néolibéralisme, expliquerait cette situation en statuant que la culture a, dès les balbutiements de la professionnalisation de l'anthropologie, constitué une "niche » marketing qui lui a permis de se distinguer des autres sciences. D'autre part, comme l'anthropologie, créée par des savants voilà plus d'un siècle dans des contextes de rapports coloniaux, aspirait à décrire les différences entre les cultures de l'Europe et des États-Unis d'Amérique et des sociétés étudiés alors, cette science est, étonnamment, "elle-même une construction hégémonique " (Saillant, Kilani et Graezer Bideau, 2011, p. 11 et p. 15). Ainsi, en cherchant l'autre à l'extérieur de soi, les anthropologues qui ne questionnent pas leur rapport au concept de culture reproduiraient la tension et la relation coloniales en s'arrogeant le pouvoir de définir l'autre à travers un système d'analyse fondé sur leurs croyances de ce qui leur apparaît être des contrastes, des écarts, et donc opèrent une différentiation où le soi, sous des dehors d'appréhension de l'autre, sert à marquer le "hors-de-soi » comme un territoire exotique à explorer pour mieux, au final, se redéfinir car exempt de contribution significative autant pour l'avancement de la connaissance que pour l'univers de provenance du collaborateur préalablement entendue entre l'anthropologue et cet autre.

La reconfiguration de la manière de traiter la culture demandée par Saillant, Kilani et Graezer Bideau (2011) mais aussi par Abu-Lughod (2005), Gupta et Ferguson (2005), de même que l'approche décentrée de la culture préconisée par Touraine (1997) pour « faire œuvre » (Le Blanc, 2009) nous amènent, si nous les acceptons, à devoir non seulement penser les réalités sociales différemment, mais aussi, comme nous l'avons mentionné, les fondations de l'anthropologie contemporaine. Par exemple, penser différemment le multiculturalisme - et l'interculturalisme n'est pas exempt d'une telle proposition sur le plan conceptuel et empirique, travail certes concrétisé avec d'autres paramètres et réalités liés à ce paradigme - exige de questionner la notion même de différence intrinsèque à la raison d'être du multiculturalisme mais aussi de l'anthropologie dont l'a priori de différence culturelle teinte l'analyse de faits sociaux, car, dans les deux cas de figure, « if we question a pre-given world of separate and discrete 'people and culture', and see instead a difference-producing set of relations, we turn from a project of juxtaposing preexisting difference to one of exploring the construction of difference in historical process » (Gupta et Ferguson, 2005, p. 614). Cette conceptualisation de la différence comme construction d'un fait social et disciplinaire, nous l'aurons maintenant compris, devrait désormais être évité pour espérer sortir du cadre restrictif qui "incarcère" les personnes étudiées par les anthropologues dans une structure dite "culturelle " qui les marginalisent du reste du monde dans l'espace-temps ainsi que spatialement par leur utilisation comme« produit de I'imagination anthropologique " (Appadurai, 1988, cité par Abu-Lughod, 2005, p. 470).

L'autre enchâssé dans une différence localisée comme socle de l'imagination anthropologique empêcherait donc la rencontre entre les personnes impliquées, quel que soit leur rôle dans la recherche et leur statut social réel ou imaginé. Cette révision du cadre de l'éthique et de la pratique anthropologique abordée sous l'angle proposé par Abu-Lughod (2005) ainsi que par Gupta et Ferguson (2005), révision et propositions actualisées par les contributions présentées par Saillant, Kilani et Graezer Bideau (2011), représentent des pistes de réflexion d'une anthropologie qui souhaite davantage traiter de la culture dans une jonction de cultures, ou d'éléments culturels qui se rencontrent, que de continuer à puiser à même les idées préconçues de la rencontre culturelle où l'autre est constamment examiné par le regard de celui qui lui confère sa différence. Ainsi pourrions-nous avancer que ces anthropologues ont réfléchi dans les débuts des années 1990, sans toutefois le nommer ainsi, au fondement de ce qui pourrait constituer une anthropologie qui transcenderait le multi-culturel (des cultures différentes qui se côtoient, celle homogénéisée des gens étudiés et celle tout autant homogénéisée des chercheurs) en s'intéressant à l'inter-culturel (des cultures différences, celles de chacune des personnes, qui vivent ensemble la recherche et créent par ce contact leur propre culture), voire même au trans-culturel (des liens qui se nouent au-delà des appartenances culturelles et qui se définissent à l'extérieur de celles-ci). Cette mouvance de l'aspect relationnel des différentes utilisations de la culture au sein des paradigmes du multi-, de l'inter- et du trans-culturel permet d'entrevoir " une typologie du contraste relationnel décroissant " d'un paradigme à l'autre, comme l'a noté Afef Benessaieh (Imbert et Benessaieh, 2010, p. 232). Au sein d'une potentielle conception transculturelle des rapports 
entre des êtres humains, il n'y a plus de chercheurs qui rencontrent les autres, ceux que les chercheurs considèrent différents d'eux, mais que des autres tous différents, aux dimensions culturelles différentes, qui se rencontrent et cherchent à établir un dialogue et du commun entre eux, c'est-à-dire de construire de nouveaux " nous ". C'est une pensée, affirme François Laplantine (2011), de "l'entre et de l'entre-deux ", qui se fraie " un chemin dans un mouvement de va-et-vient continu n'admettant aucune résolution et surtout aucune récupération ». Ce qui prime dans une telle sphère, "ce n'est pas tant celui qui va sur le terrain, ni même celui qui vient du terrain, c'est ce qui advient dans une expérience de terrain qui est une expérience du sensible » (Laplantine, 2011, p. 45).

\section{Expériences de vivre-ensemble en milieu HLM qui déhiérarchisent la « culture »}

Je ne travaille pas avec des ethnies, mais avec des personnes. Isabelle Dauplaise

Installé au cœur des Habitations Boyce-Viau, un complexe d'HLM du quartier Hochelaga-Maisonneuve à Montréal, le CJBV est un organisme d'intervention psychosociale présent depuis 1993 autant pour les 700 résidents des HLM Boyce-Viau que pour les résidents du quartier. C'est justement à titre de "voisin » (Germain et Leloup, 2006) que les intervenants et les animateurs du CJBV pratiquent une intervention de proximité: ils travaillent, en collaboration avec les résidents et différents partenaires locaux, à améliorer la qualité de vie des jeunes de 6 à 17 ans considérés comme provenant de milieux défavorisés. Le CJBV organise pour eux et leurs familles des activités favorisant une prise de pouvoir, permettant de briser l'isolement et d'avoir du plaisir. Le travail de proximité exigé par la direction du CJBV et accompli par ses employés s'établit sur la création de liens où «la confiance est au cœur de toute interaction sociale réussie » (selon le rapport annuel du CJBV, 2011, p. 2).

Le CJBV a préconisé pendant trois étés, entre autres stratégies, une approche d'intervention et d'animation s'appuyant sur la mobilisation par l'expression artistique. Des ateliers de digital storytelling ${ }^{3}$ (Truchon, 2009), un festival d'art (le Festival des arts de Boyce-Viau), la « création de ponts » avec des personnes de l'extérieur du HLM (Germain et Leloup, 2006) ainsi que l'intensification de relations de lobbying et de médiatisation de ces activités ont constitué les principaux outils de travail de cette approche, adaptée à la fois au milieu de vie au sein duquel le CJBV œuvre et aux habiletés des personnes-ressources en place pendant cette période. La conception et la mise en place de cette « infrastructure de visibilisation » ont été motivées par le constat que les personnes résidant en HLM s'exprimaient rarement par et pour elles-mêmes dans l'espace public. Nous avons donc voulu, avec la directrice du CJBV, son équipe et d'autres partenaires, offrir la possibilité aux résidents et aux personnes significatives de leur entourage de partager leurs points de vue sur leurs réalités.

\section{Le Festival des arts de Boyce-Viau, catalyseur de vivre-ensemble}

Le CJBV organise annuellement plusieurs événements qui deviennent des prétextes pour rassembler des gens aux horizons différents autour de projets communs. Le rassemblement agit comme catalyseur des redéfinitions communes et individuelles de tous les membres de ce que sont et seront, et auraient pu être, leurs vivre-ensemble au sein desquels sont constamment renégociés ses fondements par les membres en présence, quel que soit leur statut socio-économique, leur définition ethnique ou leur rôle formel et informel. Différents vivre-ensemble s'actualisent : un vivre-ensemble macro, collectif, et une multitude de micro vivre-ensemble, entre des individus au sein du collectif. L'infrastructure de visibilisation qui a été mise en place par le CJBV et ses partenaires (dont je faisais partie) constitue un de ces espaces où différents vivre-ensemble se sont déployés. Le moteur de cette infrastructure de visibilisation au sein de laquelle ont essaimé des micros et un macro vivre ensemble a été l'art. Isabelle Dauplaise (entretien personnel, 2009), alors intervenante enfance-famille au CJBV et organisatrice en chef du Festival des arts de Boyce-Viau (FABV), a souligné l'importance de l'art comme outil de mobilisation individuel et

\footnotetext{
3 Mouvement initié en Californie par le Centre for Digital Storytelling dans les années 1990, et depuis adapté et pratiqué mondialement (Hartley et McWilliam, 2009; Lambert, 2010; Lundby, 2008), le digital storytelling est une méthode qui permet à des néophytes comme à des personnes plus ferrées en nouvelles technologies médiatiques de créer de brèves histoires sur des sujets de leur choix. Ces histoires sont conçues à partir de photos, de fragments de vidéos, d'extraits musicaux et de dessins qui, généralement, appartiennent à la personne qui raconte une histoire. La particularité de ces histoires est qu'elles sont narrées au "je » et enregistrées avec la voix des personnes qui les ont réalisées. Elles constituent donc, pour plusieurs personnes qui se sentent reléguées aux marges de la société, une manière de dire "j'existe » (Bissoondath, 2007) en insérant leur voix au sein d'un espace de représentation.
} 
de groupes. : «L'art devient un élément rassembleur, tout le monde est là, oublie les différences qui sont là. Et c'est ce qui fait la beauté de la chose, les gens se mobilisent autour d'un projet. Peu importe l'âge, la différence, le background qu'ils peuvent avoir parce qu'ils ont un objectif de monter un numéro ou un vidéo pour pouvoir faire le Festival ». Elle renchérit : "La "culture" devient donc ton médium d'intervention où les barrières tombent parce que les gens sont rassemblés autour d'autres choses [que les conditions ethniques ou culturelles] ».

L'art comme élément de rassemblement a permis de créer plus de 80 films autobiographiques (digital storytelling). Ce sont principalement des des résidents des Habitations Boyce-Viau qui ont réalisé des films, mais aussi des intervenants et des animateurs du CJBV et des personnes de l'extérieur. Ces brefs films autobiographiques ainsi que des numéros de danse, de musique et des animations ont été présentés lors des trois éditions du FABV des étés 2008, 2009 et 2010. Une cinquantaine de résidents des Habitations Boyce-Viau, principalement des enfants et des adolescents mais aussi des adultes, ainsi que des personnes significatives de leur entourage, ont participé à ce festival en plein air dans les cours intérieures du complexe d’habitations, devant un public de 100 à 150 personnes, public constitué de leurs pairs, de leurs familles, d'autres résidents du HLM ainsi que de politiciens, d'autres intervenants d'organismes communautaires, de gestionnaires de programmes de subvention, de dirigeants de l'OMHM et de journalistes.

À la deuxième édition du Festival, des bulletins d'information avec trois nouvelles et capsules vidéo ont été envoyés à des partenaires des milieux communautaires, politiques, universitqires et médiatiques. Deux coffrets DVD doubles ont été produits (le premier regroupait les quelque 20 films autobiographiques de chacune des deux premières éditions du Festival et des moments forts de ce dernier et le deuxième offrait plus de 40 films autobiographiques et d'autres moments importants de la soirée de culmination de l'événement ). Pour la deuxième et troisième éditions, des partenariats ont été créés avec des gens de l'extérieur pour entretenir et développer des liens entre les résidents, ces personnes et les employés du CJBV et moi (deux activités intitulées Police Académie avec le poste de quartier 23, une activité avec des policiers de la ville de Montréal, une activité de connaissance du quartier et de la sécurité avec Tandem Mercier/Hochelaga-Maisonneuve, deux ateliers de photos en groupe avec des photographes professionnels, une séance de photos de mode avec un photographe professionnel, un atelier de tam-tam, un atelier de danse hip-hop, entre autres). Trois campagnes de relations avec les médias et de lobbying auprès des médias locaux et nationaux ainsi que des politiciens locaux et provinciaux ont également été menées (un reportage à la radio de Radio-Canada de près de 15 minutes, la nomination d'Isabelle Dauplaise à titre de Femme de l'année 2009 pour Elle Québec, la visite de Louise Harel alors députée à l'Assemblée nationale, entre autres). Bref, cette infrastructure de visibilisation a permis de mettre en valeur les résidents des Habitations BoyceViau et des personnes significatives de leur entourage car si «[c]e sont des histoires reliées à la précarité économique et sociale des HLM qui sont fréquemment véhiculées dans les medias [...] les participants de [ce] festival [ont] montr[é] leur talent et d'autres facettes de la vie en HLM » (CJBV, 2009, p. 1).

Si nous reprenons à des fins de brève analyse les sept dimensions culturelles proposées par Small, Harding et Lamont (2010), cette manière d'être et de faire détonne sur plusieurs aspects avec les perceptions habituellement entendues sur les résidents d'habitations sociales dans des espaces privés et public. Certains préjugés populaires supposent que les résidents d'habitations sociales ont comme valeur le désintérêt et possèdent un répertoire limité de stratégies d'intégration dans un engagement de longue durée. Ce projet montre le contraire par le nombre élevé de participants (une cinquantaine), dont certains se sont engagés pendant les trois années du projet. Nous avons été constamment étonnés des manières différentes de faire, de comprendre, de savoir être des participants qui inventaient leurs propres stratégies d'engagement, et nous avons accepté que c'était à nous de les recevoir dans leurs propres dynamiques. Par exemple, un participant qui a été diagnostiqué avec un retard mental léger n'arrivait pas à se souvenir de ses phrases et une jeune fille sourde et muette voulait faire un film. II était de notre devoir d'accompagner ces personnes. Dans le premier cas, nous avons écrit des bouts de phrases au tableau et dans le deuxième, nous avons travaillé également avec la jeune sœur pour traduire nos interactions. Nous avions donc comme cadre de référence un cadre où les participants pouvaient bouger et au sein duquel ils étaient accompagnés selon les besoins exprimés. Ce cadre de référence permettait également à une personne qui avait un rôle socialement circonscrit de pouvoir en occuper un autre à un moment de son choix. Par exemple, un résident est devenu aide technique pendant le Festival, une intervenante a occupé également le rôle d'organisatrice en chef du Festival, un policier est devenu un interviewé dans un film sur les gangs de rue par un des jeunes résidents et a 
organisé pendant deux étés une Police Académie, un animateur et une intervenante ont monté plusieurs des digital storytelling, un animateur a écrit et animé les trois soirées du Festival, une directrice a animé un atelier de photos et j'ai agi comme formatrice de films et coordonnatrice de projets spéciaux.

Par ailleurs, les participants de cette infrastructure de visibilisation ont réalisé des films autobiographiques dont les narrations montraient des personnes vivantes qui nous attiraient vers elles malgré les conditions de vie pouvant être perçues par les gens de l'extérieur comme étant de la pauvreté ${ }^{4}$. Ces narrations diffèrent grandement de celles véhiculant de la victimisation et qui, malheureusement, et bien qu'elles soient de moins en moins fréquentes dans l'espace médiatique, demeurent encore la stratégie nécessaire au financement de projets menés avec des personnes vivant en situation de pauvreté. Cette situation peut paraître paradoxale mais elle sert à entretenir les images de rédemption que les subventionneurs veulent des organismes travaillant auprès des personnes considérées marginales au plan sociétal (entretien avec Joëlle Dupras, 2009). Dans le même ordre d'idée, des personnes, au demeurant bien intentionnées, ont tenté, sans le vouloir, de créer des frontières symboliques en insinuant que seulement les "pauvres» devaient s'exprimer car ils n'en avaient pas souvent l'occasion contrairement aux "riches". Nous avons, au contraire, intégré toutes les personnes qui voulaient et pouvaient participer au Festival, pas seulement les personnes considérées comme "pauvres », car autrement nous aurions favorisé un régime de visibilité qui donne à voir "les pauvres petits pauvres " en cachant les "riches ", situation qui revenait à souscrire à une survisibilisation qui colonise par le regard (Beaud, Confavreux et Lindgaard, 2006).

Un des capitaux culturels de ce projet réside dans le fait que les participants appréciaient, entre autres, rire, s'amuser et produire et qu'ils étaient, de surcroît, aimés par leurs pairs et leur famille. Le rire et le plaisir sont facilement occultés des représentations des espaces marginalisés; pourtant, ces deux émotions ont été bien présentes pendant la concrétisation des films autobiographiques, les pratiques de numéros d'art pour le festival et le quotidien au complexe d'habitation. La capacité de produire des résultats de qualité est également niée par la perception populaire. Nous avons, à l'inverse, reçu plusieurs commentaires approbateurs qui allaient de «cette histoire m'a touché » à " pouvons-nous montrer quelques digital storytelling lors de l'assemblée annuelle de notre conseil d'administration? ॥. Une des questions récurrentes du projet concernait la réception des histoires biographiques. Chacune d'elles a été reçue avec respect et anticipation, de même qu'accueillie par des rires et des larmes de bonheur. Les parents étaient émus de voir leurs enfants sur grand écran et sur scène. Les invités étaient également secoués par les fragments de vie offerts par les participants. Ainsi, le FABV, à l'instar d'autres événements organisés par le CJBV, a permis l'émergence et l'arrimage de différents projets corporatifs et d'intentions individuelles. C'est grâce à ce cadre souple que des micros vivre-ensemble se sont développés pour concrétiser les objectifs et les intentions de tous les participants, ainsi que le macro vivre-ensemble nécessaire à l’organisation et la présentation de l'événement. Chacun des participants avait la possibilité de dévoiler, dans un climat de confiance, ses objectifs et ses intentions et nous avions toutes et tous la responsabilité de travailler à rassembler ceux-ci dans un tout cohérent pour chaque individu et pour l'ensemble des individus. La question de l'intentionnalité est cruciale dans une telle perspective car malgré nos objectifs respectifs. Par exemple, la direction du Centre des jeunes souhaitait une activité rassembleuse, les intervenants voulaient que les participants développent certaines habiletés, l'anthropologue espérait des données de recherche et agir selon la recommandation de Germain et Leloup (2006) en contribuant à créer des liens, les résidents demandaient à avoir du plaisir et à apprendre, les policiers espéraient être mieux accueilli sur le plan d'habitation et la direction de l'OMHM aspirait à avoir des résidents engagés dans leur milieu (car ainsi ces derniers respectent les lieux physiques et vivent de manière plus pacifique) et les résidents ambitionnaient de produire des films et des numéros de qualité qui seraient appréciés, tout en s'amusant dans un contexte d'apprentissage. Nous avions un objectif de groupe : celui de faire en sorte que nos actions respectives et communes favorisent une meilleure qualité de vie pour les résidents des HLM Boyce-Viau ainsi qu'une meilleure communication entre les résidents et les personnes qui travaillent avec eux.

\footnotetext{
4 Les entrevues réalisées dans le cadre des mes études doctorales avec une trentaine de résidents vivant en HLM montrent que ces derniers ne se considèrent pas, en majorité, comme « pauvres ». Ce sont les regards externes qui apportent ce sentiment d’être différent et « se libérer » de ce regard est vital dans ce contexte (McAll et collab, 2001).
} 


\section{Pédagogie de l'effort, pas de l'ethnicité}

L'un des éléments qui a permis la réussite de cette démarche de création de multiples micro et macro vivreensemble activant de nouvelles compréhensions et relations entre des participants, des partenaires et des organisateurs de ce projet a été la relégation de l'aspect culturel ou ethnique à un niveau secondaire, pour faire place à un objectif de formation. "On ne fait pas une fixation sur une religion ou une ethnicité », nous a confié Isabelle Dauplaise lors d'un entretien. "C'est plus pédagogique", a ajouté Véronique Morissette, alors intervenante au CJBV. Dans les modèles de réussite proposés, le transfert d'attention s'effectue de l'ethnicité vers l'effort que chacun d'entre nous devons effectuer pour réussir, peu importe qui nous sommes et d'où nous venons.

Cette manière de faire, on l'a vécu, entre autres, avec Jonas, notre professeur de danse pour le Festival, a expliqué Isabelle Dauplaise. Jonas est d'origine haïtienne et réussit en danse. [...] Les enfants participaient à son atelier pour apprendre la danse dans le but de monter un spectacle au festival, et il a su faire œuvre de pédagogue en disant: " moi, j'ai réussi. Et pourquoi j'ai réussi? C'est parce que j'ai fait des efforts, j'ai découvert que j'avais envie de faire ça, danser, et qu'il fallait que j'aille à l'école, que je travaille, que je pratique ». II n’a pas établi sa pédagogie autour du fait qu'il était un " Haïtien » qui avait réussi.

En reprenant, là aussi, les dimensions culturelles proposées par Small, Harding et Lamont (2010), nous constatons que la valeur prédominante est l'effort, le cadre de référence est l'idéologie néolibérale et le répertoire s'inscrit dans une dynamique de développement de ses habiletés selon ses goûts. La narration s'inspire de l'adage populaire " quand on veut, on peut ". La frontière symbolique s'arrime au départage de ce que nous aimons, des actions qu'il faut entreprendre pour réussir et de la foi en nos capacités à concrétiser notre rêve, le capital culturel étant cette capacité de rêver. Le cadre institutionnel est représenté ici par la société néolibérale au sein de laquelle ces jeunes vivent, tout comme les intervenants et Jonas, ainsi que par le Centre des jeunes Boyce-Viau qui met en place cette structure d'apprentissage pouvant être réflexive si certains le souhaitent. Cette manière d'appréhender la vie peut paraître naïve, voire reproductrice des barrières systémiques et structurelles qui empêchent certaines personnes de notre société de pouvoir s'émanciper, mais le pari est de fournir des outils et des espaces pour leur permettre de manœuvrer dans cette société néolibérale, et de soutenir, au besoin, cette incursion. Les intervenants du CJBV, les spécialistes embauchés et les experts externes inscrivent leurs actions dans cette direction, sans nier l'environnement au sein duquel ils agissent, ni les difficultés structurelles, relationnelles et de représentations auxquelles ils doivent faire face.

\section{La culture fait partie de l'individu, mais n'est pas l'individu}

La directrice, les intervenants et les animateurs du CJBV ainsi que les diverses personnes offrant leurs services/expertises (dont moi) considéraient, inséraient et négociaient l'aspect culturel d'une manière singulière dans leur travail et leurs rapports avec les résidents aux diverses origines de Boyce-Viau : aucune intervention psychosociale, aucune animation et aucune activité ne ciblaient de manière frontale les origines ethniques ou les appartenances culturelles réelles ou imaginées des personnes qui fréquentaient le CJBV. Cette manière de travailler était directement reliée aux convictions de Joëlle Dupras, alors directrice du CJBV. Elle ne croyait pas aux projets de mixité qui priorisaient des activités de dévoilement de sa culture et de son ethnicité comme éléments d'intégration sociale, projets du type des soupers à vocation culturelle ou ethnique. Sa philosophie touchait toutes les actions du CJBV, notamment le FABV et les partenariats développés pendant l'organisation du Festival.

Quand il est abordé de manière transversale et non de manière frontale, le concept de culture offre des capacités épistémologiques et méthodologiques nouvelles, et beaucoup plus prometteuses. Joëlle Dupras nous en livre un aperçu lorsqu'elle développe sa vision de la personne :

L'origine culturelle de quelqu'un fait partie de la personne. [...] Quand on parle de travailler avec les origines ethnoculturelles ou les minorités visibles, c'est qu'on perçoit leur origine ethnoculturelle ou leur parcours migratoire comme étant quelque chose de parallèle à leur soi. [...] Ce qu'il faut, c'est questionner cette personne-là [pour la connaître]. C'est inévitable alors que son origine ethnoculturelle ou son parcours migratoire va entrer en ligne de compte, mais pas de façon parallèle à soi, mais de manière intégrée à une globalité plus vaste. [...] Ton parcours migratoire fait partie de toi, ta dépendance aux narcotiques fait partie de toi, le fait que tu sois mère fait partie de toi. [...] Ton travail, quand tu fais de l'intervention, c'est d'intervenir avec une personne à part entière. 
Joëlle Dupras illustre ici que la culture, ou des aspects culturels comme les sept dimensions culturelles de Small, Harding et Lamont, sont des morceaux d'une personne, des morceaux qui diffèrent d'une personne à une autre, et qui fluctuent au cours d'une vie. Pour accéder à ces fragments distincts d'une personne à l'autre, ce n'est donc pas simplement l'activité qui compte, mais autant comment, par qui et dans quelle(s) intention(s) cette activité est organisée. La « voie ", la « sphère citoyenne » anticipée par Gérard Bouchard et Charles Taylor (2008), ainsi que l'apprentissage de la diversité qu'ils pensent nécessaires à l'avenir du Québec, ne peuvent se concrétiser s'il n'y a pas d'espaces de vivre-ensemble où naissent amitiés et conflits, et ces situations ne sont possibles qu'autour d'un projet commun plus vaste, où l'expression individuelle de tous sera reconnue à travers un groupe. C'est ce que nous avons entrepris de réaliser avec cette infrastructure de visibilisation, qui a permis l'organisation et la présentation d'un festival d'arts, d'ateliers de digital storytelling, de relations de lobbying et avec les médias ainsi que d'activités de consolidation et de création de liens.

\section{Conclusion : tendre vers le transculturel}

On ne connaît la culture qu'à travers les cultures. Edgar Morin

L'individu ne s'oppose pas tellement à la collectivité, en soi; c'est individuel et collectif qui s'opposent en chacun de nous comme deux parties différentes de l'âme.

Gilles Deleuze

J'ai tenté de montrer qu'en démythifiant et en repositionnant la hiérarchisation de la culture comme un des éléments constitutifs d'un individu ou d'un groupe, et non comme l'entité fondamentale de laquelle découlent les éléments constitutifs d'un individu ou d'un groupe, conception fréquente autant dans l'imaginaire populaire, médiatique qu'universitaire, nous encourageons de nouvelles manières d'entrer en relation, stimulant ainsi l'établissement de situations de vivre-ensemble plus axées sur des facteurs concrets qui rassemblent et dissocient les personnes nées à l'extérieur du Canada et celles nés au Canada que sur des images reliées aux ethnicités qui seraient en présence les unes pour les autres et vice-versa. Pour ce faire, j'ai opté, en tant qu'anthropologue, pour une posture critique du concept de culture et je me suis inspirée d'un cadre théorique qui évite de focaliser l'attention sur celui-ci, bien qu'il reste essentiel à la compréhension de la recherche présentée ici.

Le cas de l'infrastructure de visibilisation mise en œuvre par le CJBV et ses partenaires, dont l'anthropologue signataire de ce texte, s'avère intéressante à plusieurs niveaux. Cette infrastructure, bien qu'elle ait été implantée dans un espace spatialisé comme milieu de pauvreté, ce qui est encore malheureusement perçu dans l'espace populaire comme une possible "culture ", n’a jamais été pensée comme un programme à vocation culturelle qui pourrait réformer les gens. Cette infrastructure a reconnu dès ses origines et à plusieurs moments de son existence qu'elle mettait en relation des gens aux pouvoirs sociaux, politiques et économiques et de représentation différents. Au sein de cette infrastructure, et par celle-ci, nous, organisateurs, avons donc tenté de déstabiliser ces inégalités de pouvoir en nous positionnant chacun également comme des participants qui réalisent et présentent publiquement leur digital storytelling, animent les soirées, aident les "gars de tech ", font de la course pendant Police Académie, entre autres. Ainsi, nous n'avons pas éradiqué la " culture " mais l'avons traitée différemment, d'une part, en insistant sur le comportement et l'environnement au sein duquel ce comportement s'effectue, et d'autre part, en nous commettant nous aussi dans cet espace représentationnel avec les résidents et les personnes significatives de leur entourage. Bref, cette infrastructure de visibilisation, institutionnalisée mais se voulant souple pour répondre à plusieurs attentes de prime abord divergentes, est un exemple concret de situation où ont été réunis des gens aux dimensions culturelles différentes dans des expériences vécues ensemble et où nous avons eu, pour quelques mois, un projet de vie commun (Touraine, 1997, p. 234). Nous avons donc fait œuvre de notre vie où « le social est appréhendé depuis la « créativité de l’agir » (Le Blanc, 2009, p. 117) ».

Ce qui m’apparaît le plus concluant de ces expériences de vivre-ensemble en milieu HLM, c'est qu'en décentrant la culture nous avons, chacun dans nos sphères professionnelles et individuelles, pensé autrement ce que signifie vivre et travailler dans un milieu étiqueté comme "de pauvreté ». Au lieu de tenir pour acquises les différences culturelles véhiculées sur diverses tribunes populaires et professionnelles, nous avons exploré de

Alterstice - Revue Internationale de la Recherche Interculturelle, vol. $1, n^{\circ} 1$ 
manière empirique la construction de nos différences et ressemblances à un moment de nos histoires individuelles et communes. Aussi bien les personnes vivant en milieu de pauvreté que celles travaillant avec elles ont pris le risque de sortir du "cadre qui incarcère » (Gupta et Ferguson, 2005), en favorisant une jonction d’éléments culturels.

Afin de ne pas contribuer à forger et disséminer la différence qui se construit sur et se reproduit par l'acceptation du concept de cultures différenciées, celle de l'anthropologue et celle des gens étudiés, fondement sur lequel s'est édifiée l'anthropologie et fondement contesté par certains anthropologues (Abu-Lughod, 2005; Graezer Bideau, 2011; Saillant 2011), c'est donc une expérience de " recherche impliquée " (Fletcher et Cambre, 2009) qui m'a permis de se joindre à plusieurs autres personnes pour vivre ce « qui advient dans une expérience du terrain " (Laplantine, 2011, p. 45). Ces apparitions relationnelles se font et se défont dans un mouvement perpétuel d'aller et retour entre des rencontres du multiculturel, de l'interculturel ${ }^{5}$ et du transculturel. Pour reprendre les distinctions de Benessaieh, le multiculturel apparaît comme "la simple juxtaposition du différent culturel ", l'interculturel, comme "une interaction dualiste ou polarisante ", et le transculturel, comme "une relationnalité mobile et constante de référents culturels » (Imbert et Benessaieh, 2010, p. 231-232).

Le transculturel inquiète les chercheurs car sa définition ne peut être que fluide, toujours à refaire et défaire et surtout, le transculturel implique, comme je l'ai expliqué, " des modifications importantes au sujet de l'identitaire comme de la notion [...] de " culture » " (Imbert et Benessaieh, 2010, p. 232). Tendre vers l'option du transculturel m’apparaît sensé dans le contexte social actuel. Pour y parvenir, il faut travailler autant le culturel que le structurel, et réfléchir de manière différente l'économie morale du travail auprès des personnes vivant en situation de pauvreté. Comme l'explique Andrea Riccardi (2007),

[r]éfléchir sur la réalité consiste [...] à avoir la patience de se confronter à elle [...] et apprendre à opérer des distinctions [...] pour éviter de créer des monstres ou des chimères dans le laboratoire de notre pensée, en classant dans la même catégorie des manifestations ou des expressions différentes » (p. 164).

Or, et Hannah Arendt (1970/1996) l'entrevoyait déjà,

Les clichés, les phrases toutes faites, l'adhésion à des codes d'expression ou de conduites conventionnels et standardisés, ont socialement la fonction reconnue de nous protéger de la réalité, de cette exigence de pensée que les événements et les faits éveillent en vertu de leur existence » (p. 26-27).

Tel est notre défi, réfléchir aux réalités sociales de manière différente pour mieux agir sur ces réalités, et non pervertir ces réalités en agissant sur des images de ce qu'elles seraient. Poursuivre la réflexion et le dialogue entamés ici et ailleurs sur le multiculturel, l'interculturel et le transculturel fait partie des moyens qui s'offrent aux chercheurs. J'ai tenté d'esquisser dans ce texte, qu'à la lueur des expériences de terrain vécues en milieu HLM à Montréal, l'option du transculturel me semble la plus prometteuse pour le type de pratique impliquée que je privilégie.

\section{Remerciements}

Je remercie Yvan Leanza pour sa patience et ses suggestions judicieuses. Des remerciements chaleureux vont également à ma directrice de thèse, Francine Saillant, ainsi qu'à Joëlle Dupras, Isabelle Dauplaise et Véronique Morissette. Notons qu'il est fréquent de conserver l'anonymat des informateurs des recherches ethnographiques,

\footnotetext{
5 La notion d'interculturalisme, qui n'est jamais devenue une Loi ou une politique au Québec, se serait développée en opposition à la politique du multiculturalisme (Rocher et collab, 2007). Or la ligne démarquant les deux concepts s'avère plutôt mince. Dans les faits, la notion d'interculturalisme est enchâssée de manière implicite dans les politiques d'intégration et d'aménagement de la diversité au Québec, sans être toutefois clairement articulée comme l'est le multiculturalisme canadien, qui a fait l'objet d'une politique et d'une loi spécifique (Rocher et collab, 2007), une lacune qui devrait être comblée selon les coprésidents de la Commission de consultation sur les pratiques d'accommodement reliées aux différences culturelles (CCPARDC), Gérard Bouchard et Charles Taylor (2008). Ces derniers proposent que " I'interculturalisme québécois a) institue le français comme langue commune des rapports interculturels; b) cultive une orientation pluraliste soucieuse de la protection des droits; c) préserve la nécessaire tension créatrice entre, d'une part, la diversité et, d'autre part, la continuité du noyau francophone et le lien social; d) met[tte] un accent particulier sur l'intégration et la participation et e) préconise la pratique des interactions " (p. 121).
} 
mais que dans certaines circonstances il est nécessaire de contrecarrer cette règle, notamment quand les personnes souhaitent témoigner à visage découvert afin d'être reconnues. C'est le cas de ces trois intervenantes du CJBV pendant ma recherche.

Mes travaux ont été financés par le CRSH, le FQRSC et le CÉLAT. Le CJBV a financé l'infrastructure de visibilisation mise en place aux Habitations Boyce-Viau de laquelle sont tirées les analyses partielles présentées dans cet article.

Les activités ont été rendues possibles grâce à la participation de Pierre Boudreau, Suzanne Duhaime, Karinne Henri, Claude Lizotte, Pierre Manning, Serge Villandré et Jonas.

\section{Références bibliographiques}

Abu-Lughod, L. (2005). Writing against Culture. Dans H. Moore et T. Sanders (dir.), Anthropology in Theory. Issues in Epistemology (p. 466-479). Oxford : Blackwell Publishing.

Arendt, H. (1970/1996). Considérations morales. Paris : Éditions Payot et Rivages.

Armony, V. (2007a). Le Québec expliqué aux immigrants. Montréal : VLB Éditeur.

Armony, V. (2007b). Mémoire présenté à la Commission de consultation sur les pratiques d'accommodement reliées aux différences culturelles. Mémoire récupéré le 10 août 2011 sur le site de la Commission de consultation sur les pratiques d'accommodement reliées aux différences culturelles:

http://www.accommodements.qc.ca/documentation/memoires/Montreal/armony-victor-memoirepresente-a-la-commission-de-consultation-sur-les-pratiques-d-accommodement-reliees-aux-differencesculturelles-montreal.pdf

Beauchemin, J. et Bock-Côté, M. (dir.). (2007). La cité identitaire. Montréal : Athéna Éditions.

Beaud, S., Confavreux, J. et Lindgaard, J. (2006). La France invisible. Paris : La Découverte.

Benessaieh, A. (2010). Multiculturality, Interculturality and Transculturality. Dans A. Benessaieh (dir.), Amériques transculturelles/Transcultural Americas (p. 11-38). Ottawa : Presses de l'Université d'Ottawa.

Bissoondath, N. (2007). L'âge de la confession. Moncton : Goose Lane Editions et Université de Moncton.

Bock-Côté, M. (2009). L'identité occidentale du Québec ou l'émergence d'une « cultural war » à la québécoise. Recherches sociographiques, 50(3), 537-570.

Bock-Côté, M. (2007). La dénationalisation tranquille. Montréal : Boréal.

Bouchard, G. et Taylor, C. (2008). Fonder l'avenir. Le temps de la conciliation. Québec : Gouvernement du Québec.

Centre des jeunes Boyce-Viau. (2009). Communiqué : Le Festival des arts de Boyce-Viau : Une cinquantaine de résidents d'un HLM de Montréal et des personnes significatives de leur entourage montrent qui ils sont.

Fassin, D. (2009). Les économies morales revisitées. Annales. Histoire, sciences sociales, 6, 1237-1266.

Ferraro, G. et Andreatta, S. (2010). Cultural Anthropology. An Applied Perspective. Belmont : Wadsworth CENGAGE Learning.

Fletcher, C. et Cambre, C. (2009). Digital Storytelling and Implicated Scholarship in the Classroom. Journal of Canadian Studies, 43(1), 1-22.

Frerer, K. et Vu, C. (2007). An anthropological View of Poverty. Journal of Human Behavior in the Social Environment, 16(1), 73-86.

Germain, A. et Leloup, X. (2006). II était une fois dans un HLM... Portrait de l'intervention communautaire dans quatre HLM de type " plan d'ensemble » de l'arrondissement Mercier-Hochelaga-Maisonneuve. Récupéré le 10 août 2011 du site du Centre des Jeunes Boyce-Viau : http://cjbv.com/pdf/II_etait_une_fois_dans_un_HLM.pdf 
Graezer Bideau, F. (2011). Culture. Dans F. Saillant, M. Kilani et F. Graezer Bideau (dir.), Manifeste de Lausanne. Pour une anthropologie non hégémonique (p. 53-55). Montréal : Liber.

Gupta, A. et Ferguson, J. (2005). Beyond «Culture »: Space, Identity, and the Politics of Difference. Dans H. Moore et T. Sanders (dir.), Anthropology in Theory. Issues in Epistemology (p. 608-617). Oxford : Blackwell Publishing.

Hartley, J. et McWilliam, K. (dir.). (2009). Story Circle. Digital Storytelling Around the World. Oxford : WileyBlackwell.

Imbert, P. et Benessaieh, A. (2010). Conclusion : la transculturalité relationnelle. Dans A. Benessaieh (dir.), Amériques transculturelles/Transcultural Americas (p. 231-242). Ottawa : Presses de l'Université d'Ottawa.

Kroeber, A. et Kluckhohn, C. (1952). Culture: A critical Review of Concepts and Definitions. Papers of the Peabody Museum of American Archeology and Ethnology, 47(1).

Kymlicka, W. (2007a). Ethnocultural Diversity in a Liberal State: Making Sense of the Canadian Model(s). Dans K. Banting, T. Courchene et F. Seidle (dir.), Belonging? Diversity, Recognition and Shared Citizenship in Canada (p. 39-86). Montreal : The Institute for Reseach on Public Policy (IRPP).

Kymlicka, W. (2007b). Multicultural Odysseys: Navigating the New International Politics of Diversity. Oxford : Oxford University Press.

Kymlicka, W. (2007c). Tester les limites du multiculturalisme libéral? Le cas des tribunaux religieux en droit familial. Éthique publique, 9(1), 27-39.

Lachapelle, G. (dir.). (2008). Diversité culturelle, identités et mondialisation : de la ratification à la mise en œuvre de la convention sur la diversité culturelle. Sainte-Foy : Presses de l'Université Laval.

Lambert, J. (2010). Digital Storytelling: Capturing Lives, Creating Community. Berkeley : Life on the Water Inc.

Laplantine, F. (2011). Connaissance réciproque. Dans F. Saillant, M. Kilani et F. Graezer Bideau (dir.), Manifeste de Lausanne. Pour une anthropologie non hégémonique (p. 44-46). Montréal : Liber.

Le Blanc, G. (2009). L'invisibilité sociale. Paris : Presses Universitaires de France.

Lende, D. (2010a [18 octobre]). The Culture of Poverty Debate. Récupéré le 25 juillet 2011 du blogue Plotsblogs : http://blogs.plos.org/neuroanthropology/2010/10/18/the-culture-of-poverty-debate

Lende, D. (2010b [26 octobre]). The Culture of Poverty Debate Continued. Récupéré le 25 juillet 2011 du blogue Plotsblogs : http://blogs.plos.org/neuroanthropology/2010/10/26/the-culture-of-poverty-debatecontinued/

Lisée, J.- F. (2007a). Nous. Montréal : Boréal.

Lisée, J.- F. (2007b). Pour un nouvel équilibre entre tous les « Nous » québécois. Document récupéré le 14 août 2011 sur le site de la Commission de consultation sur les pratiques d'accommodement reliées aux différences culturelles https://www.accommodements.qc.ca/documentation/memoires/A-N-Montreal/lisee-jeanfrancois.pdf

Lundby, K. (dir.). (2008). Digital Storytelling, Mediatized Stories. Self-representations in New Media. New York : Peter Lang Pub.

McAll, C. et collab. (2001). Se libérer du regard : agir sur la pauvreté au centre-ville de Montréal. Montréal : Éditions Saint-Martin.

Office municipal d'habitation de Montréal. (2011). Boyce-Viau. Récupéré le 5 août 2011 du site de l'OMHM Un toit où évolue la famille : http://www.omhm.qc.ca/node/391/description/643

Riccardi, A. (2007). Vivre ensemble. Paris : Desclée De Brouwer.

Rocher, F. et collab. (2007). Le concept d'interculturalisme en contexte québécois : généalogie d'un néologisme. Document récupéré le 10 août 2011 sur le site de la Commission de consultation sur les pratiques 
d'accommodement reliées aux différences culturelles:

http://www.accommodements.qc.ca/documentation/rapports/rapport-3-rocher-francois.pdf

Saillant, F. (2011) Différence et différend. Dans F. Saillant, M. Kilani et F. Graezer Bideau (dir.), Manifeste de Lausanne. Pour une anthropologie non hégémonique (p. 59-61). Montréal : Liber.

Saillant, F., Kilani, M. et Graezer Bideau, F. (dir.). (2011). Manifeste de Lausanne. Pour une anthropologie non hégémonique. Dans F. Saillant, M. Kilani et F. Graezer Bideau (dir.), Manifeste de Lausanne. Pour une anthropologie non hégémonique (p. 9-35). Montréal : Liber.

Small, M., Harding, D. et Lamont M. (2010). Reconsidering Culture and Poverty. The ANNALS of the American Academy of Political and Social Science, 629, 6-27.

Small, M., Lamont, M. et Harding, D. (2011, 15 août). A Fresh Approach to Culture. Récupéré le 18 août 2011 du site Spotlight on poverty : http://www.spotlightonpoverty.org/ExclusiveCommentary.aspx?id=7aacf39a-9a80474a-8cad-a4238f19a4af

Taché, P. (2009). La culture québécoise et ses références identitaires : une ethnographie de la francisation à l'école de langues de l'Université Laval (ÉLUL). Mémoire de maîtrise non publié, Université Laval.

Touraine, A. (1997). Pourrons-nous vivre ensemble? : Égaux et différents. Paris : Fayard.

Truchon, K. (2009). Montrer l'autre, faire apparaître notre relation à l'autre. Le digital storytelling pour raconter «l'Africanité » au sein d'un HLM de Montréal. Ethnologie, 31(2), 191-217. 